\title{
Pediatric Convulsive Status Epilepticus: Act Fast, No matter With What!
}

\author{
PAMOHAMMEd KUNJU ${ }^{1 *}$ ANd H AHAMEd SUBIR ${ }^{2}$ \\ ${ }^{1}$ Department of Pediatric Neurology, Medical College Trivandrum; and ${ }^{2}$ Department of Neurology, MES Medical College, \\ Perinthalmanna; Kerala.*drpamkunju@gmail.com
}

$\mathrm{C}$ onvulsive status epilepticus (SE) is associated with considerable mortality and morbidity [1], and irreversible brain damage may occur if timely treatment is not given [2]. The International League Against Epilepsy (ILAE) has included two time frames in its definition of SE viz, time to intervene $(\mathrm{T} 1=5 \mathrm{~min})$ and time of occurrence of permanent brain damage $(\mathrm{T} 2>30 \mathrm{~min})$ [2]. So early and effective treatment that can achieve the above goal is searched for by many researchers, and the last decade has witnessed many studies that have compared various drugs and different routes. Many protocols also have incorporated those new drugs arbitrarily [3,4].

Though for the acute (first stage) seizure control benzodiazepines are the main treatment, superiority of lorazepam is challenged by many studies and current conclusion is that intramuscular midazolam and intravenous diazepam are as effective as intravenous lorazepam [5].However, a consensus has not yet been achieved regarding which is the best antiepileptic in the armamentarium for the rapid control of the seizures once the benzodiazepines fail (stage 2). Levetiracetam, sodium valproate, phenytoin/fosphenytoin, lacosamide, and phenobarbital are being widely used and extensively studied for this indication [4]. A third stage is recognized as refractory SE, defined as SE persisting despite sufficient dose of benzodiazepines and at least one antiepileptic drug (AED), irrespective of time. Midazolam, propofol, and thiopental are used in an ICU setting to manage this stage but with limited support of evidence from wellcontrolled trials. Finally, a fourth stage is also recognized super refractory SE defined as SE, that continues for 24 hours or more after the use of anesthetic therapy. Management of this stage is still hazier, with anecdotal treatment modalities like ketamine, IV immunoglobulin, ketogenic diet, and surgical measures [6].

Since standardized management of second stage can bring about realistic positive outcomes, evidence-based recommendations are required for drug-selection. Extrapolation of results from Western studies to the
Indian context may not be appropriate given the differences in the body constitution and the genetic diversity, which may affect both drug dosages and drug metabolism. In this issue of Indian Pediatrics, Vignesh, et al. [7] report on their well-designed study comparing the efficacy of phenytoin, valproate and levetiracetamfor the management of pediatric CSE. They showed a better seizure control with levetiracetam (94\%), phenytoin $(89 \%)$, and valproate $(83 \%)$ when compared to other studies that had used much higher dosages. Three facts are highlighted by this study [7].Firstly, there is equal efficacy of all drugs, meaning thereby that we need not confine ourselves to phenytoin as the sole second line drug. Moreover, a lower dose is enough for seizure control, and such a lower dose is associated with lesser chances of adverse reactions.

In a randomized multicentric trial (ESSET trial), Kapur, et al. [8] found that the same three AEDs (fosphenytoin instead of phenytoin) were effective in approximately half the patients with benzodiazepinerefractory convulsive SE, and they did not differ significantly with regard to safety. However, that study used a higher dosage of levetiracetam $(60 \mathrm{mg} / \mathrm{kg})$ and valproate $(40 \mathrm{mg} / \mathrm{kg})$ instead of uniform $20 \mathrm{mg} / \mathrm{kg}$ used by the present study [7]. Similarly, EcLiPSE trial [9] showed that levetiracetam had comparative efficacy to phenytoin and suggested that former could be an appropriate alternative to phenytoin. But, the ConSEPT trial concluded that levetiracetam is not superior to phenytoin for second-line management of pediatric convulsive SE [10]. It may be noted that all the three trials showed an effectiveness of only $50 \%$ in children as compared to $>80 \%$ response in the present study [7]. However, the uniform infusion time (20 min) taken for blinding by Vignesh, et al. [7] compared to $10 \mathrm{~min}$ and $5 \mathrm{~min}$ infusion by other trials might have impacted the time for seizure control by valproate and levetiracetam, which are safer to be infused faster. As the rapid control of seizure will bring a better outcome, this advantage could have been better utilized by modifying the blinding technique. Another 
meta-analysis by Yaziry, et al. [11] concluded that valproate, levetiracetam and phenobarbital can all be used as first line therapy in benzodiazepine-resistant SE but did not support the use of phenytoin.

The fact that the dose used in this study for levetiracetam $(20 \mathrm{mg} / \mathrm{kg})$ and sodium valproate $(20 \mathrm{mg} / \mathrm{kg})$ was well below the internationally recommended dose but with better outcome suggests that regional variation in dose will improve the outcome and reduce adverse effects while using these options. The methodology of random sequence generation and allocation concealment increases the power of this study but at the same time points to the fact that the three drugs have different infusion rates which haveimportant implications for seizure control.Levetiracetam can be administered more rapidly (5$10 \mathrm{~min}$ ) than phenytoin (20min), which could potentially terminate convulsive status epilepticus faster with levetiracetam than phenytoin. This factor would not be taken into account during allocation concealment thus affecting the good outcome in both levetiracetam and valproate arm as they would be infused slowly at the same rate as phenytoin.Moreover, the ease of drug preparation and administration favours the newer antiepileptics rather than phenytoin. This study; however, has not provided the important epidemiological insights that could help in making the AED choice, as the seizure control and outcome may depend more on the etiology than the drug used.

We feel that this study has clearly established the equal effectiveness and safety of the three drugs. We need to generate more evidence, and experiment with dosing and act quickly with the effective medications we have, so as to bring out region-based guidelines taking into consideration the epidemiological and socioeconomic aspects.

Funding: Nil; Competing interest:None stated.

\section{REFERENCES}

1. Smith DM, McGinnis EL, Walleigh DJ, Abend NS. Management of status epilepticus in children. J Clin Med.
2016;5:47.Available from: https://www.mdpi.com/20770383/5/4/47/htm. Accessed on February 8, 2020.

2. Trinka E, Cock H, Hesdorffer D. A definition and classification of status epilepticus - Report of the ILAE Task Force on Classification of Status Epilepticus. Epilepsia. 2015;56:151523.

3. Glauser T, Shinnar S, Gloss D, Alldredge B, Arya R, Bainbridge J,et al.Evidence-Based Guideline: Treatment of Convulsive Status Epilepticus in Children and Adults: Report of the Guideline Committee of the American Epilepsy Society.Epilepsy Curr. 2016;16:48-61.

4. Mishra D, Sharma S, Sankhyan N, Konanki R, Kamate M, Kanhere S, et al. Consensus Guidelines on Management of Childhood Convulsive Status Epilepticus. Indian Pediatr. 2014;51:975-90.

5. McTague A, Martland T, Appleton R.Drug management for acute tonic-clonic convulsions including convulsive status epilepticus in children.Cochrane Database Syst Rev. 2018;1:CD001905.

6. Dubey D, Kalita J, Misra UK. Status epilepticus: Refractory and super-refractory. Neurol India. 2017;65 Supp1:S12-7.

7. VigneshVM, Kumar RR, Mahadevan S. Comparison of phenytoin and valproate and levetiracetam in pediatric convulsive status epilepticus: A randomized double-blind controlled clinical trial. Indian Pediatr. 2020;57:222-7.

8. Kapur J, Elm J, Chamberlain JM, Barsan W, Cloyd J, Lowenstein $\mathrm{D}$, et al. Randomized trial of three anticonvulsant medications for status epilepticus. N Engl J Med. 2019;381:2103-13.

9. Lyttle MD, Rainford NEA, Gamble C, Messahel S, Humphreys A, Hickey H, et al; Paediatric Emergency Research in the United Kingdom \& Ireland (PERUKI) collaborative. Levetiracetam versus phenytoin for secondline treatment of paediatric convulsive status epilepticus (EcLiPSE): A multicentre, open-label, randomised trial. Lancet. 2019;393:2125-34.

10. Silbergleit R, Elm JJ.Levetiracetam no better than phenytoin in children with convulsive status epilepticus. Lancet.2019;393:2101-2.

11. Yasiry Z,Shorvon SD. The relative effectiveness of five antiepileptic drugs in treatment of benzodiazepineresistant convulsive status epilepticus: A meta-analysis of published studies. Seizure. 2014;23:167-74. 